3 may 2005

\title{
On Lie bialgebras of loops on orientable surfaces
}

\author{
Attilio Le Donne ${ }^{1}$ \\ Dipartimento di Matematica, Universitá di Roma "La Sapienza" , Piazzale \\ Aldo Moro 1, I-00185, Roma, Italia
}

\begin{abstract}
Goldman (Invent. Math. 85(2) (1986) 263) and Turaev (Ann. Sci. Ecole Norm. Sup. (4) 24 (6)(1991) 635) found a Lie bialgebra structure on the vector space generated by non-trivial free homotopy classes of loops on an orientable surface. Chas ( Combinatorial Lie bialgebras of curves on surfaces, Topology 43 (2004) 543), by the aid of the computer, found a negative answer to Turaev's question about the characterization of multiples of simple classes in terms of the cobracket, in every surface of negative Euler characteristic and positive genus. However, she left open Turaev's conjecture, namely if, for genus zero, every class with cobracket zero is a multiple of a simple class. The aim of this paper is to give a positive answer to this conjecture.
\end{abstract}

MSC: primary 57M99, secondary 17B62.

Keywords: Surfaces; Homotopy classes; Lie bialgebras.

\section{Introduction.}

In 1989, Turaev [3] introduced for an oriented surface $\Sigma$, a Lie cobracket in the module $Z(\Sigma)$ generated by the homotopy classes of non-trivial loops on $\Sigma$.

The cobracket complements the Goldman Lie bracket $[2]$ in $Z(\Sigma)$ and makes it a Lie bialgebra in the sense of Drinfeld.

\footnotetext{
${ }^{1}$ Tel.: +3906 49913249; fax: +39 0644701007

E-mail address: ledonne@mat.uniroma1.it
} 
Every multiple of a simple class has cobracket zero, so Turaev [4] asked whether it is true that if the cobracket is zero then the class is a multiple of a simple class.

Chas [1], giving a combinatorial presentations of the Lie bialgebra by reduced cyclic words, found, by the aid of the computer, that in every surface of negative Euler characteristic and positive genus there exist classes with cobracket zero which are not multiples of simple classes.

Always with the aid of the computer, she checked that Turaev's characterization holds for words of length up to sixteen on the pair of pants (i.e. a two sphere with three punctures) so that she suggested that it is possible for genus zero surfaces.

Here we answer affirmatively this conjecture by showing that:

In a genus zero surface every class with cobracket zero is a multiple of a simple class.

\section{Curves and loops}

Let $\Sigma$ be a smooth oriented surface, possibly with boundary.

The circle $S^{1}$ is oriented counterclockwise, this will make all curves oriented.

Given two points $\alpha, \beta$ of the circle $S^{1}$, we denote with $\alpha \beta$ (resp. $-\alpha \beta$ ) the oriented embedded arc in $S^{1}$ which starts at $\alpha$ (resp. $\beta$ ), going in the positive, i.e., counterclockwise (resp. negative, i.e., clockwise) direction and terminates at $\beta$ (resp. $\alpha$ ), so in $\alpha \beta$ (resp. $-\alpha \beta$ ) we consider this order. If $\alpha=\beta, \alpha \beta$ (resp. $-\alpha \beta$ ) will be all $S^{1}$ (resp. $-S^{1}$ ) with counterclockwise (resp. clockwise) direction. Note that $-\alpha \beta$ is different from $\beta \alpha$, even if both start at $\beta$ and end at $\alpha$.

A curve in $\Sigma$ will be a piecewise smooth map $f$ from an arc $\alpha \beta$ to $\Sigma$. If $f(\alpha)=f(\beta)$ the curve is a loop.

Two homotopic curves $f$ and $g$ are said also equivalent, and we write $f \cong g$. A loop is trivial if it is null-homotopic.

From now on, $f$ will be a curve.

If the arc $a b$ is a subset of the domain of $f$, then $f_{a b}$ will be the restriction of $f$ to $a b$.

For two curves $f_{\alpha \beta}$ and $g_{\gamma \delta}$, so that $f(\beta)=g(\gamma), f_{\alpha \beta} \cdot g_{\gamma \delta}$ is the usual composition of curves. 
$f_{-\alpha \beta}$ will be the inverse curve of $f_{\alpha \beta}$. ( It start at $f(\beta)$ and end at $f(\alpha)$ ).

For two equivalent curves $f$ and $g$ we put $g \prec f$ if $i m(g) \subset i m(f)$. (Note that we can have $g \prec f$ also if $f$ and $g$ have not the same domain).

Denote with Class $(f)$ (resp. RClass $(f)$ ) the class of all $g$ homotopic to $f$ (resp. $g$ such that $g \prec f$ ).

\section{Double and crossing points}

The point $p$ is a double point if $f^{-1}(p)=\{x, y\}$, with $x \neq y$; in this case we put $x \approx y$.

If $f(a)$ is a double point, we call $a$ a double point too.

We consider only curves with a finite number of double points and no other multiple point.

Moving the curve in $V$ will mean use a homotopy that leaves all point not in $V$ fixed.

A double point $p$ is a crossing point if, for some neighborhood $V$ of $p$, we cannot eliminate the double point moving the curve in $V$.

We denote $\operatorname{Dbl}(f)$ (resp. Cross $(f)$ ) the set of double (resp. crossing ) points of $f$, and $\sharp D b l(f)$ and $\sharp C r o s s(f)$ their numbers.

An $f$ is generic if it is smooth and has only transversal (i.e., with transversal tangents) double points; (hence each double point is crossing, i.e., $\sharp D b l(f)=\sharp C r o s s(f))$.

We say that $f$ is minimal if it has the minimal number of crossing points in $R \operatorname{Class}(f)$, ( i.e., $\sharp C r o s s(f) \leq \sharp C r o s s(g)$ for each $g$ such that $g \prec f$ ).

The Feynmann diagram of $f_{\alpha \beta}$ is formed by the arc $\alpha \beta$ and all the straight segments $e_{p}=e_{a b}$, if $a<b$ in $\alpha \beta$, having endpoints the two points $a, b$ of $f^{-1}(p)$, for each double point $p$ of $f$. We say that two different double points $p, q$ are linked if $e_{p} \cap e_{q} \neq \emptyset$.

We say that a Feynmann diagram is linked if there are two double points which are linked.

\section{Monogons, bigons and trigons}

A straight segment $e_{a b}$ of the Feynmann diagram of $f$ forms a monogon if $f_{a b}$ is a null-homotopic loop.

Two linked straight segments $e_{a b}$ and $e_{c d}$ of the Feynmann diagram, with $a<c<b<d$, form a direct bigon of $f$ if $f_{a c} \cong f_{b d}$. 
Two non-linked straight segments $e_{a b}$ and $e_{c d}$ of the Feynmann diagram, with $a<c<d<b$, form a inverse bigon of $f$ if $f_{a c} \cong f_{-b d}$.

A direct bigon is a particular kind of a trigon.

Two linked straight segments $e_{a b}$ and $e_{c d}$ of the Feynmann diagram, with $a<c<b<d$, form a trigon of $f$ if $f_{b d} \cdot f_{c b} \cdot f_{a c} \cong f_{a c} \cdot f_{c b} \cdot f_{b d}\left(=f_{a d}\right)$.

\section{Lemma 1.}

Every generic minimal non-trivial $f$ has neither monogons nor bigons or trigons.

Proof. Let $f=f_{\alpha \beta}$.

If $e_{a b}$ is a monogon of $f$ then put $g=f_{\alpha a} \cdot f_{b \beta}$.

Let $\alpha \leq a<b<c<d \leq \beta$.

If $e_{a b}$ and $e_{c d}$ form an inverse bigon of $f$ then put $g=f_{\alpha a} \cdot f_{-c d} \cdot f_{b c} \cdot f_{-a b} \cdot f_{d \beta}$. If $e_{a b}$ and $e_{c d}$ form direct bigon or a trigon of $f$ then put $g=f_{\alpha a} \cdot f_{c d}$. $f_{b c} \cdot f_{a b} \cdot f_{d \beta}$.

In all cases, $g \prec f$ but $\sharp$ Cross $(g)<\sharp \operatorname{Cross}(f)$. Contradiction. $\diamond$

Let $\psi$ be a simple loop in $\mathbb{R}^{2}, D_{\psi}$ the bounded component of $\mathbb{R}^{2} \backslash \psi$, and $p_{1}, \ldots, p_{n} \in D$. ( We say that a curve is inside $\psi$ if his image is in $\operatorname{cl}\left(D_{\psi}\right)$ ).

Put $\Omega(\psi)=\operatorname{cl}\left(D_{\psi}\right) \backslash\left\{p_{1}, \ldots, p_{n}\right\}=\left(D_{\psi} \cup \psi\right) \backslash\left\{p_{1}, \ldots, p_{n}\right\}$

\section{Lemma 2.}

Let $f: \alpha \beta \longrightarrow \Omega(\psi)$ be a generic minimal curve with $m$ double points so that:

1. $f(\alpha) \in i m(\psi)$,

2. $\alpha$ and $\beta$ are not double points of $f$,

3. Each simple loop $f_{a b}$ is homotopic to $\psi$ in $\Omega(\psi)$.

Then there are $a_{i}, b_{i} \in \alpha \beta$, for each $i \leq m$, such that:

- $\alpha<a_{m}<\ldots<a_{1}<b_{1}<\ldots<b_{m}<\beta$, and $a_{i} \approx b_{i}$, for $i \leq m$, (i.e., $f$ has a non linked Feynman diagram),

- $\sigma_{i+1}=f_{a_{i} a_{i+1}} \cdot f_{b_{i+1} b_{i}}$, for $i<m$, and $\sigma_{1}=f_{a_{1} b_{1}}$ are simple equivalent loops (and hence homotopic to $\psi$ ), 
- $\sigma_{i}$ is inside $\sigma_{i+1},\left(i m\left(\sigma_{i}\right) \cap i m\left(\sigma_{i+1}\right)=\left\{f\left(a_{i}\right)\right\}=\left\{f\left(b_{i}\right)\right\}\right)$, for $i<m$; $f(\beta)$ is outside $\sigma_{m}$.

Hence $f_{a_{i} b_{i}} \cong \psi^{i}$, for $i \leq m$ ( see fig. 1$)$

\section{Figure 1}

\section{Figure 2}

\section{Proof}

The proof is by induction on $m$.

If $m=1$ the only thing to demonstrate is that $f(\beta)$ is outside $f_{a_{1} b_{1}}$. But, if it were not so, the double point $f\left(a_{1}\right)=f\left(b_{1}\right)$ would not be a crossing point. (see fig. 2)

Let us assume the lemma true for $m$ and $f$, with $m+1$ crossing points, verifying the hypothesis of lemma.

Choose $\delta \notin D b l(f)$ so that in $\delta \beta$ there is only one double point that we call $b_{m+1}$ and let $h=f_{\alpha \delta}$.

Clearly $\sharp D b l(h)=m$ and $h$ verifies the three properties of lemma and so there are $a_{i}, b_{i} \in \alpha \delta$ for each $i \leq m$, such that

- $\alpha<a_{m}<\ldots<a_{1}<b_{1}<\ldots<b_{m}<\delta$, and $a_{i} \approx b_{i}$, for $i \leq m$,

- $\sigma_{i+1}=f_{a_{i} a_{i+1}} \cdot f_{b_{i+1} b_{i}}$, for $i<m$, and $\sigma_{1}=f_{a_{1} b_{1}}$ are simple equivalent loops,

- $\sigma_{i}$ is inside $\sigma_{i+1}$, for $i<m ; f(\delta)$ is outside $\sigma_{m}$.

Since in in $\delta \beta$ there is the only double point $b_{m+1}$ of $f$, we have that $f_{\delta \beta}$ cannot meet $\sigma_{i}$ for $i<m$ and so $f(\beta)$ is outside $\sigma_{m}$ or between $\sigma_{m}$ and $\sigma_{m-1}$.

Let $a_{m+1}$ be such that $a_{m+1} \approx b_{m+1}$.

So we have one of the following cases:

1. $a_{m+1} \in \alpha a_{m}$

2. $a_{m+1} \in a_{m} a_{m-1} \quad\left(a_{2} \in a_{1} b_{1}\right.$, if $\left.m=1\right)$

3. $a_{m+1} \in b_{m-1} b_{m} \quad$ ( case not considered if $m=1$ )

4. $a_{m+1} \in b_{m} \delta$ 


\section{Figure 3}

\section{Figure 4}

\section{Figure 5}

\section{Figure 6}

Put $e_{i}=e_{a_{i} b_{i}}$, for $i \leq m+1$.

In case 2), $e_{m}$ and $e_{m+1}$ would form a direct bigon, (see fig. 4); in fact $f_{a_{m} a_{m+1}} \cong f_{-b_{m} b_{m+1}}$.

In case 3), $e_{m}$ and $e_{m+1}$ would form a trigon, (see fig. 5), because $f_{a_{m} a_{m+1}}$. $f_{a_{m+1} b_{m}} \cdot f_{b_{m} b_{m+1}} \cong f_{b_{m} b_{m+1}} \cdot f_{a_{m+1} b_{m}} \cdot f_{a_{m} a_{m+1}}$; in fact $\left(f_{a_{m} a_{m+1}} \cdot f_{a_{m+1} b_{m}}\right)$. $\left(f_{b_{m} b_{m+1}} \cdot f_{a_{m+1} b_{m}}\right) \cong\left(f_{b_{m} b_{m+1}} \cdot f_{a_{m+1} b_{m}}\right) \cdot\left(f_{a_{m} a_{m+1}} \cdot f_{a_{m+1} b_{m}}\right)$, since their images are between $\psi$ and $\sigma_{1}$ and the fundamental group of a circular crown is commutative.

In case 4 ), $e_{m+1}$ would be a monogon, (see fig. 6).

In case 1 ), if $\sigma_{m+1}=f_{a_{m+1} a_{m}} \cdot f_{b_{m} b_{m+1}}$ were null homotopic then we would have an inverse bigon; otherwise $\sigma_{m+1} \cong \psi$.

It is still to be proved that $f(\beta)$ is outside $\sigma_{m}$. But, as in case $m=1$, if $f(\beta)$ were inside $\sigma_{m}$, then the double point $f\left(a_{m+1}\right)=f\left(b_{m+1}\right)$ would not be a crossing point, (see fig 3 ). $\diamond$

\section{The main result}

\section{Theorem}

In a genus zero surface $\Sigma$ every free homotopy class of loops with cobracket zero is a multiple of a simple class.

Proof. Hence $\Sigma=S^{2} \backslash\left\{p_{1}, \ldots, p_{k}\right\}$ is a two sphere with $k$ punctures; clearly if $k<3$ every class is a multiple of a simple class.

Let $\Phi$ be a non simple class of loops on $\Sigma$, and $\varphi: S^{1} \longrightarrow \Sigma$ be a minimal generic representative of $\Phi$.

Every $\varphi_{a b}$ which is a simple loop devides the sphere $S^{2}$ into two disks and so $\left\{p_{1}, \ldots, p_{k}\right\}$ into two proper parts. ( $\varphi$ cannot contain monogons since it is minimal ! )

Among them, choose $\varphi_{\bar{a} \bar{b}}$ so that one of the two parts in which $\left\{p_{1}, \ldots, p_{k}\right\}$ is partioned is a minimal subset. We can assume that this part is $\left\{p_{1}, \ldots, p_{n}\right\}$. Put $\Psi=\operatorname{Class}\left(\varphi_{\bar{a} \bar{b}}\right)$ 
Since the cobracket of $\Phi$ is

$$
\Delta(\Phi)=\Delta(\varphi)=\sum_{a \approx b} \operatorname{sign}(a, b) \cdot \operatorname{Class}\left(\varphi_{a b}\right) \otimes \operatorname{Class}\left(\varphi_{b a}\right)
$$

where $\operatorname{sign}(a, b)=1$ if the couple of the positive tangent direction of $\varphi$ at $a$ and the positive tangent direction of $\varphi$ at $b$ is a positive pair according to the orientation of $\Sigma$; otherwise $\operatorname{sign}(a, b)=-1$. ( Remember that the definition of $\operatorname{sign}(a, b)$ can be given also for each loop and that the cobracket is independent from the chosen representative of $\Phi)$.

Since $\Delta(-\varphi)=-\Delta(\varphi)$, we can assume that $\varphi_{\bar{\alpha} \bar{\beta}}$ goes around $\left\{p_{1}, \ldots, p_{n}\right\}$ counterclockwise.

So there is a couple $(a, b)$ with $a \approx b$ such that Class $\varphi_{a b}=\Psi$

We will show that, if $\Phi$ is not a multiple of the simple class $\Psi$, then for each couple $(a, b)$ with $a \approx b$ such that Class $\varphi_{a b}=\Psi$ is $\operatorname{sign}(a, b)=-1$.

\section{Figure 7}

\section{Figure 8}

Since the free non-trivial homotopy classes of loops are free generators of the Lie bialgebra, this will show that $\Delta(\Phi) \neq 0$.

Hence, ab absurd, let us suppose that $a \approx b$ with Class $\varphi_{a b}=\Psi$ and $\operatorname{sign}(a, b)=1$.

Now, putting $p_{k}=\infty, \Sigma=\mathbb{R}^{2} \backslash\left\{p_{1}, \ldots, p_{k-1}\right\}$ and $\varphi_{a b}$ is a loop in $\mathbb{R}^{2}$ going around $p_{1}, \ldots, p_{n}$ counterclockwise.

If all $\operatorname{im}(\varphi)$ is inside $\varphi_{a b}$ then, choosing $\alpha$ and $\beta$ with $\alpha<b<a<\beta$ and putting $f=\varphi_{\alpha \beta}$ we have that $f$ verifies the property of lemma 2 with $\psi=\varphi_{a b}$.

Hence, if $f$ has $m$ double points, in the notations of lemma, we have that $a_{m}=b, b_{m}=a$ and that $f_{a_{m} b_{m}}=\varphi_{b a} \cong \psi^{m}$. Hence $\varphi \cong \psi^{m+1}$, contrarily to hypothesis.

Then suppose that not all $i m(\varphi)$ is inside of $\varphi_{a b}$. Since $\operatorname{sign}(a, b)=1$ we have the situation of figure 8, i.e., there is $c$ and $d$ so that $c<a<b<d$ with $\varphi_{c a}$ and $\varphi_{b c}$ inside of $\varphi_{a b}$. Choose the arc $c d$ maximal with this property. Then $c$ and $d$ are double points and $\varphi(c), \varphi(d) \in i m\left(\varphi_{a b}\right)$.

Let $\psi$ be a simple loop, $\psi \cong \varphi_{a b}$ with $\operatorname{im}\left(\varphi_{a b}\right) \subset D_{\psi}$ so that not all $\operatorname{im}(\varphi)$ is inside $\psi$, and $i m(\psi)$ contains no double point of $\varphi$.

Take $s<c<d<t$ such that $\varphi(s), \varphi(t) \in i m(\psi)$ and $i m\left(\varphi_{s t}\right) \subset D_{\psi}$. 
Hence $f=\varphi_{s t}$ verifies the property of lemma 2 for $\psi$.

Again, in the notations of lemma, we have that $a_{1}=a, b_{1}=b$, and in $\varphi_{a b}=\varphi_{a_{1} b_{1}}$ there would be, by the lemma, no double point of $f$ exept $f(a)=f(b)$, but $f(c)$ and $f(d)$ are. A contradiction. $\diamond$

\section{References}

[1] Chas, Moira, Combinatorial Lie bialgebras of curves on surfaces, Topology 43 (2004) 543-568

[2] Goldman, William M., Invariant functions on Lie groups and Hamiltonian flows of surface group representations. Invent. Math. 85 (1986), no. $2,263-302$

[3] Turaev, Vladimir G., Algebras of loops on surfaces, algebras of knots, and quantization, Braid Groups, Knot Theory and Statistical Mechanics, Adv.Ser.Math.Phys.,vol.9, World Sci.Publishing, Teaneck,NJ,1989, 59-95.

[4] Turaev, Vladimir G., Skein quantization of Poisson algebras of loops on surfaces. Ann. Sci. Ecole Norm. Sup.(4) 24(1991), no.6 , 635-704 


\section{Figure Captions}

fig. 1. Lemma $2, m=3$

fig. 2. $\mathrm{m}=1, f(\beta)$ inside $f_{a_{1} b_{1}}$

fig. 3. Case (1), $\mathrm{m}+1=3, f(\beta)$ inside $\sigma_{m}$

fig. 4. Case (2), $m+1=4$

fig. 5. Case (3), $m+1=4$

fig. 6. Case (4), $m+1=4$

fig. 7. $\operatorname{sign}(a, b)=-1$

fig. 8. $\operatorname{sign}(a, b)=1$ 


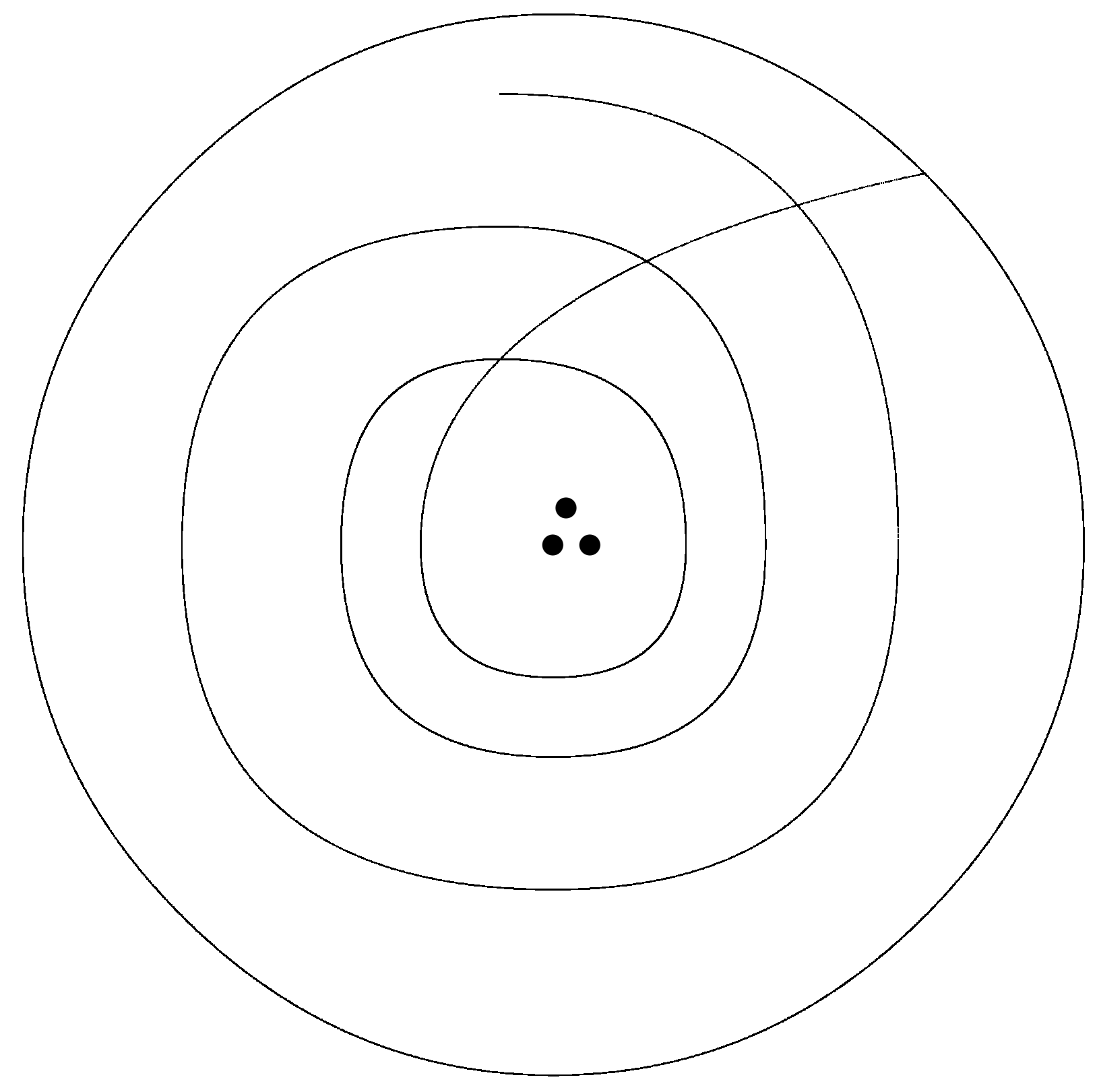

fig. 1 


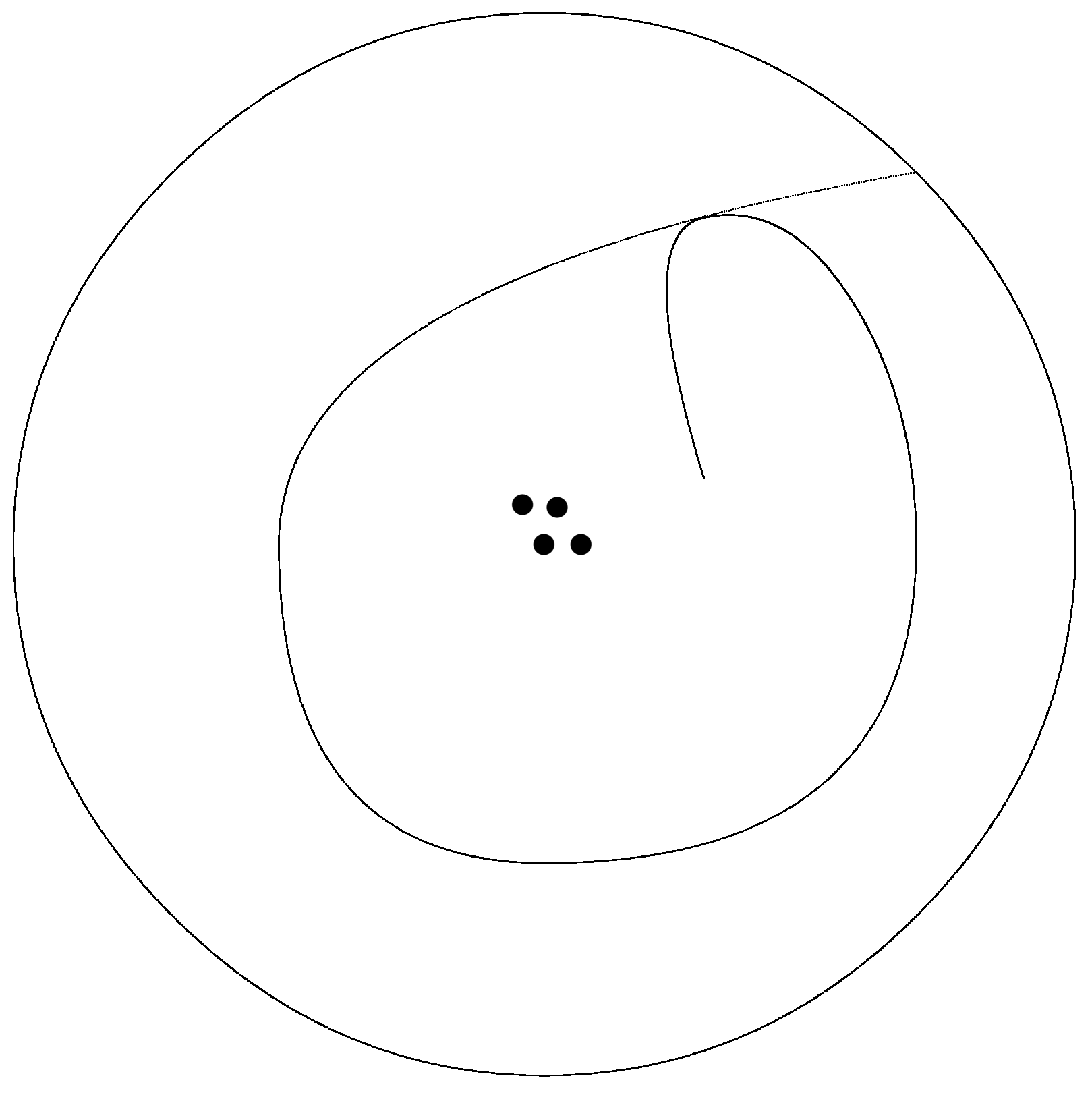

fig. 2 


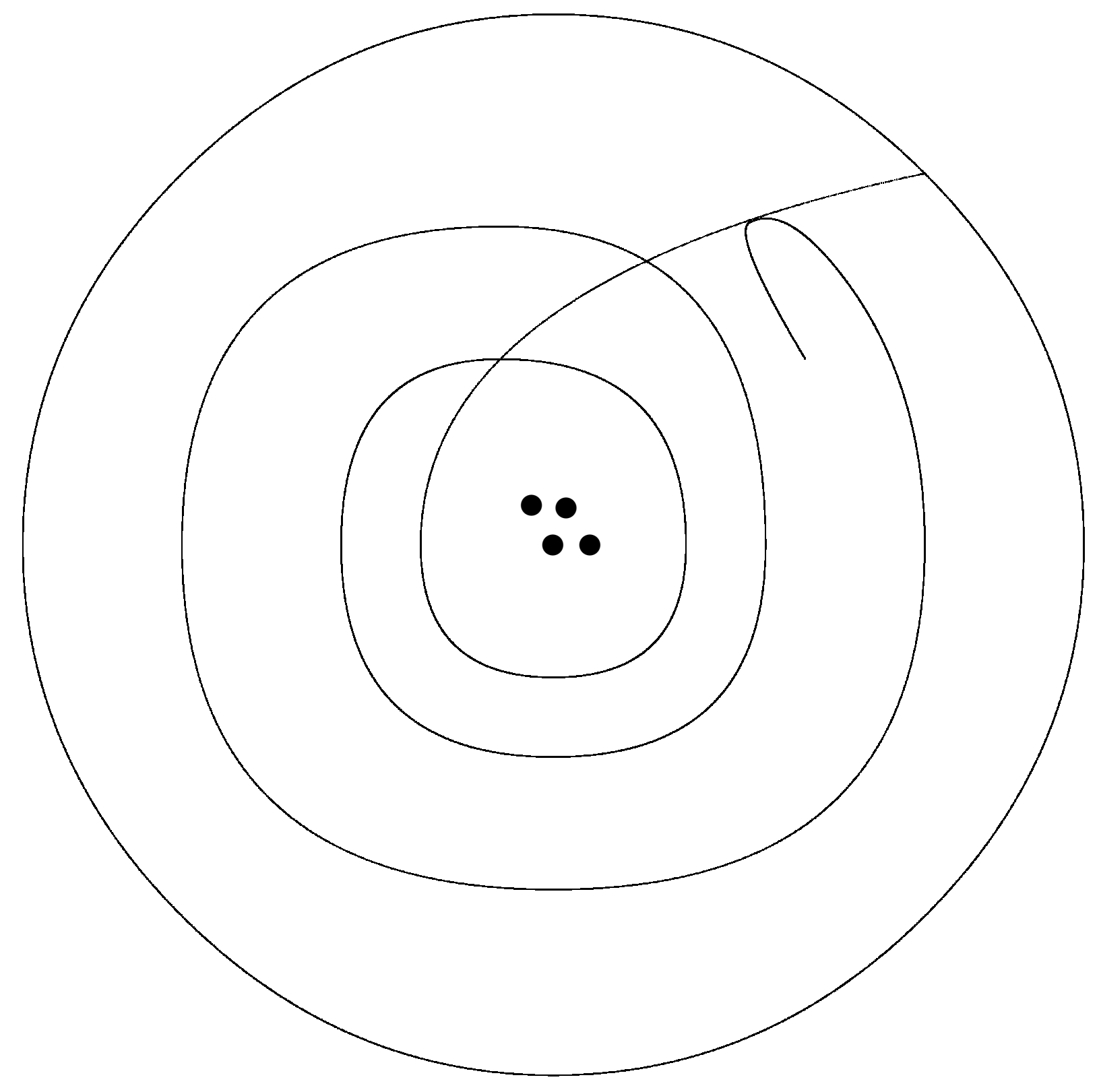

fig. 3 


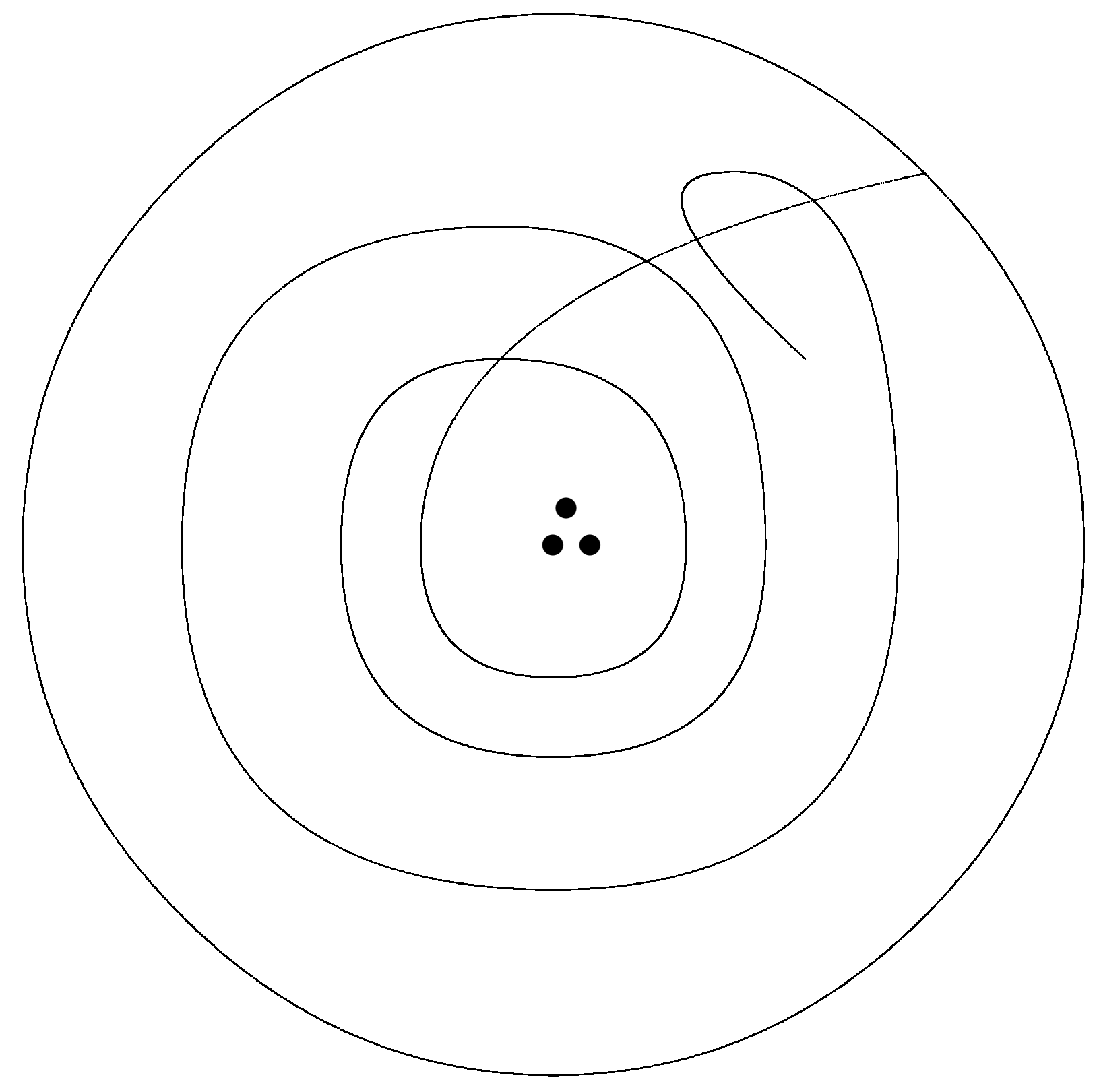

fig. 4 


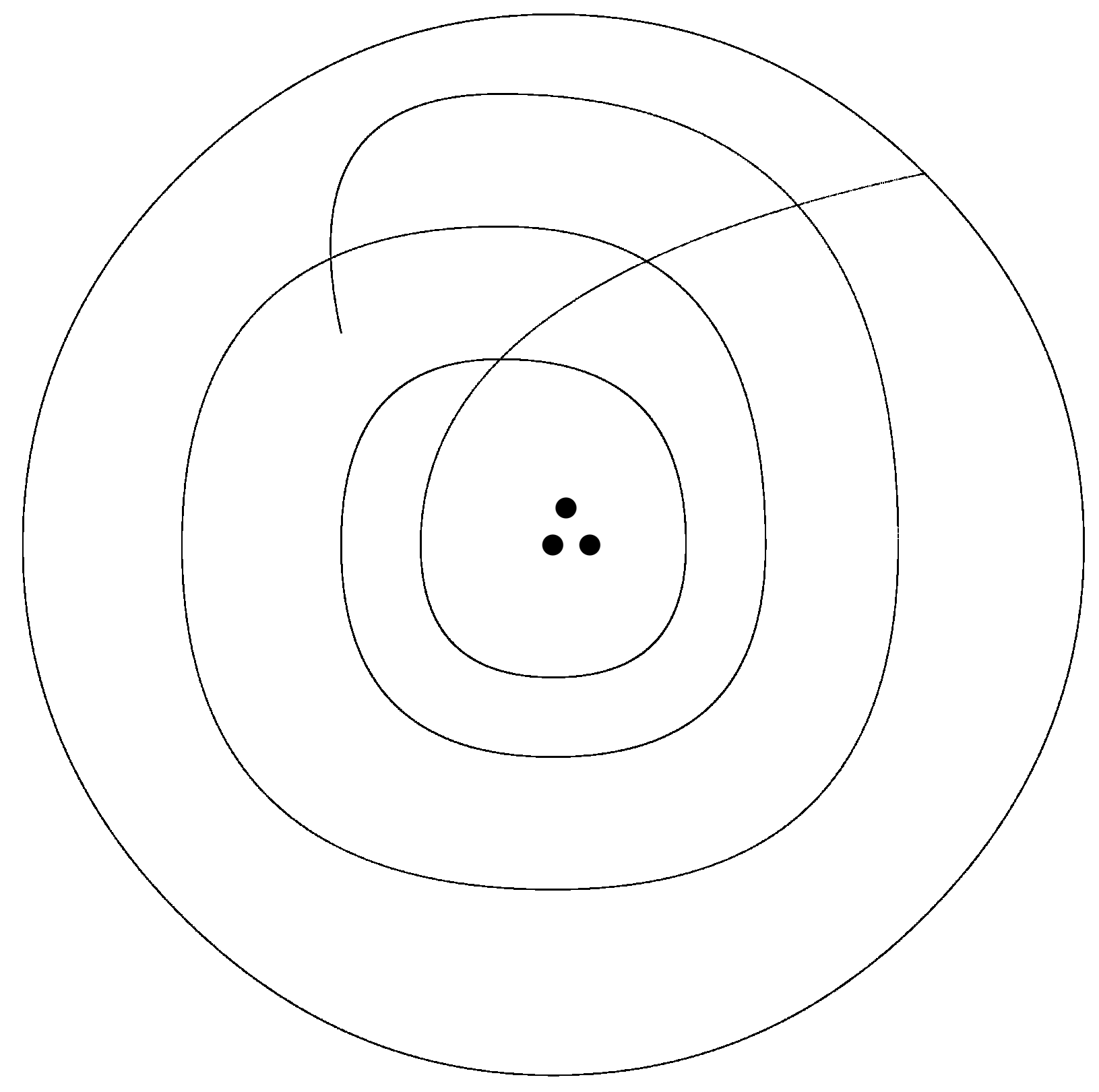

fig. 5 


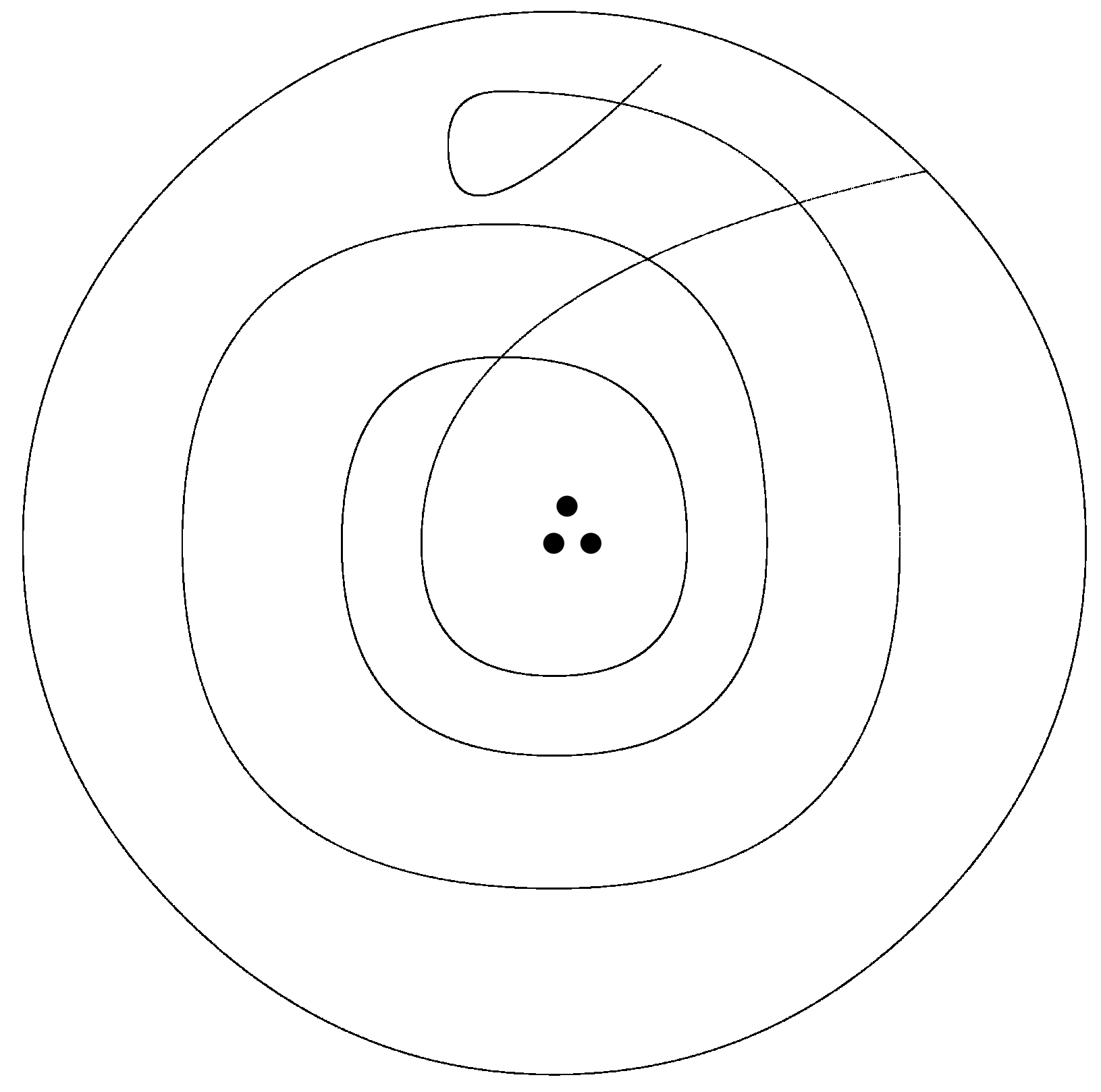

fig. 6 


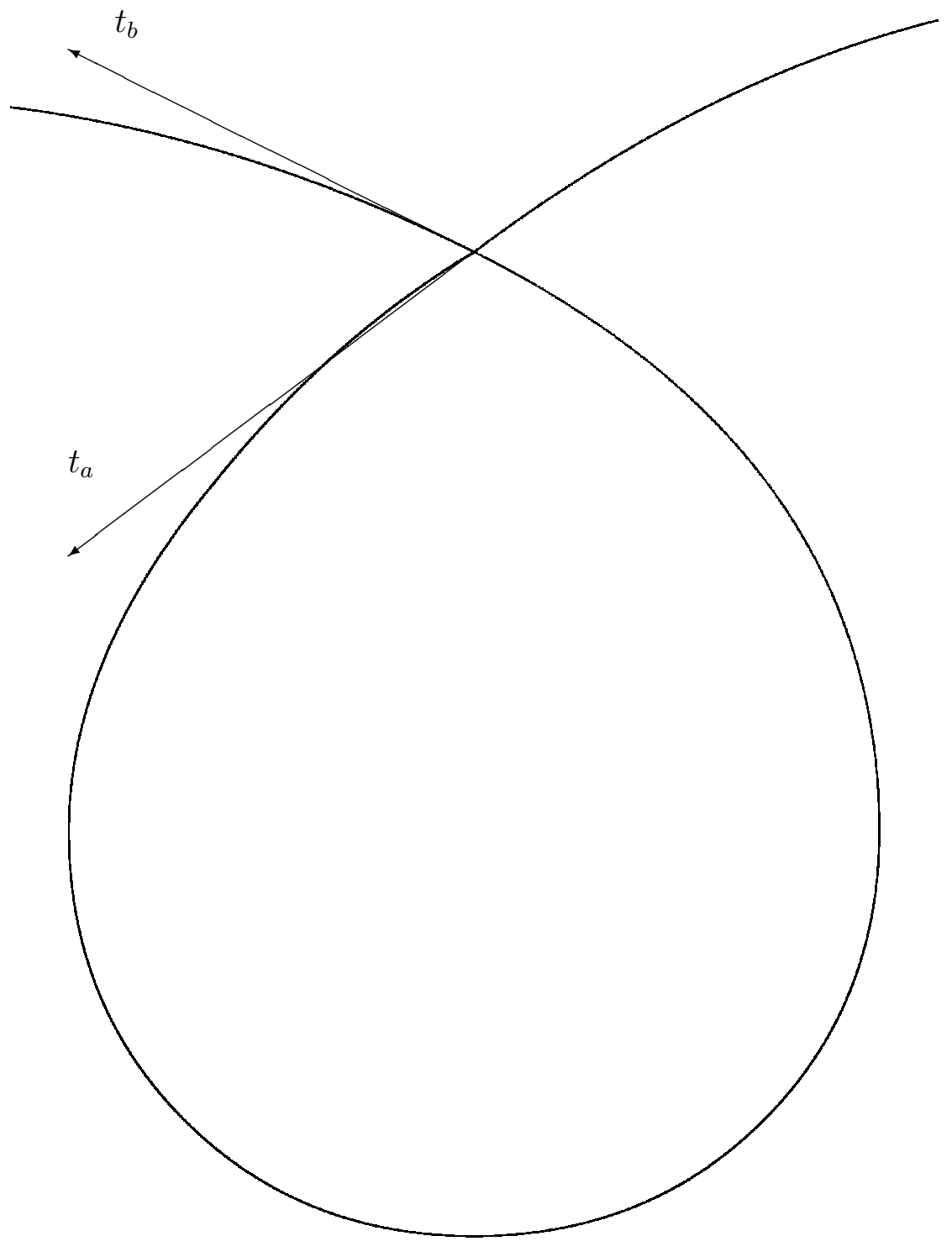

fig. 7 


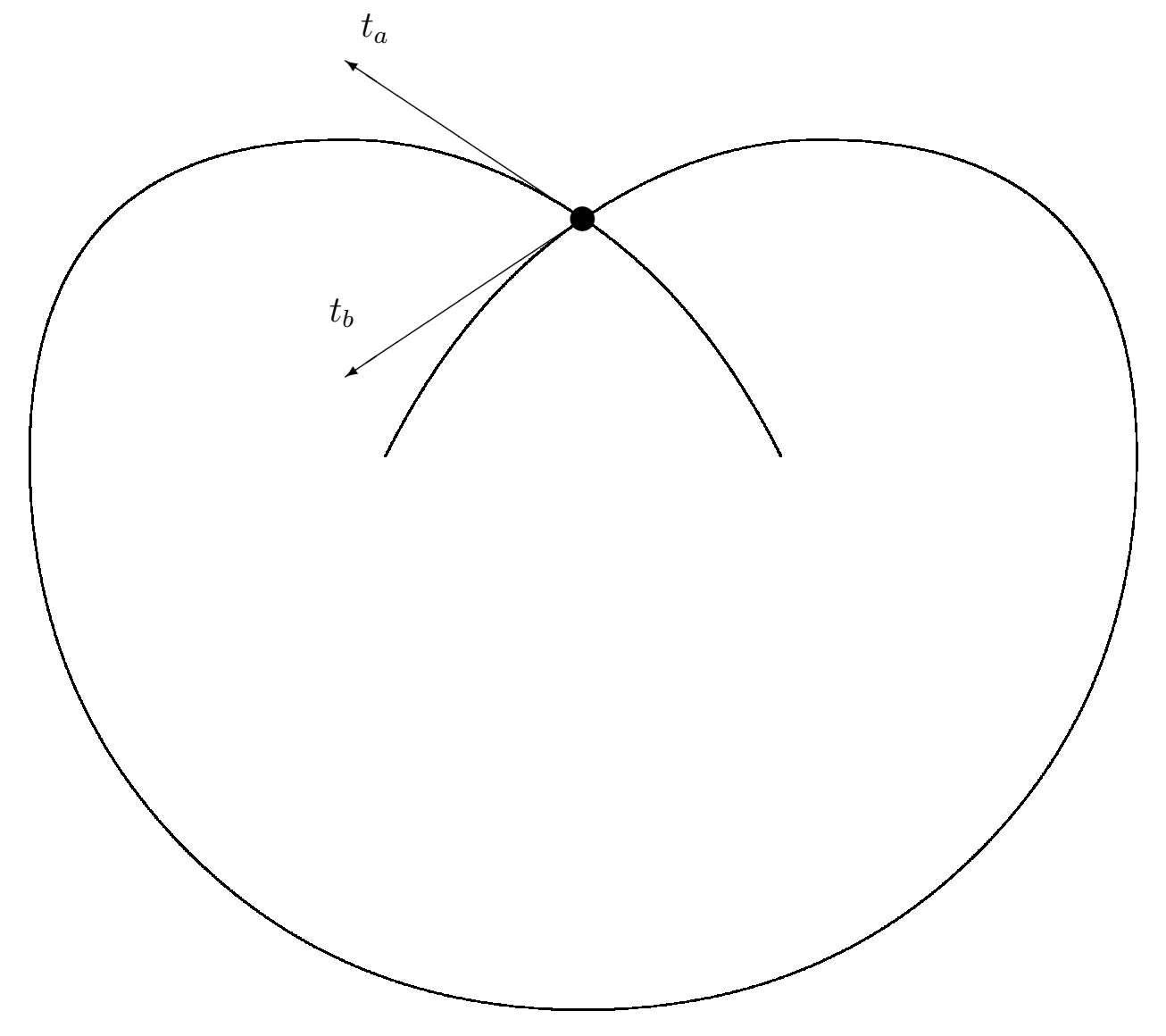

fig. 8 